\title{
Precision Kaon Physics with KLOE
}

\author{
Flavio Archilli* on behalf of KLOE collaboration: \\ Dipartitimento di Fisica e INFN Sezione di "Tor Vergata", Rome, Italy \\ E-mail: flavio.archilli@roma2.infn.it
}

\begin{abstract}
A $\phi$-factory offers the possibility to select pure kaon beams: neutral kaons from $\phi \rightarrow K_{S} K_{L}$ are in fact produced in pairs and the detection of a $K_{S}\left(K_{L}\right)$ tags the presence of a $K_{L}\left(K_{S}\right)$,the same holds for charged kaons. This allows us to perform precise measurement of kaon properties. The KLOE collaboration has measured most decay branching ratios of $K_{S}, K_{L}$ and $K^{ \pm}$mesons together with the $K_{L}$ and the $K^{ \pm}$lifetime, and the shape of the form factors involved in kaon semileptonic decays. These results provide the basis for the determination of the CKM parameter $V_{U S}$ and the most precise test of the unitarity of the quark flavor mixing matrix.

Bounds on new physics extensions of the standard model with lepton flavor violation have been set using the KLOE result on $R_{K}=\Gamma(K e 2) / \Gamma(K \mu 2)$ based on the complete data set of $2.2 \mathrm{fb}^{-1}$. The final $1.3 \%$ accuracy on the ratio $R_{K}$ has been achieved measuring the differential width $d \Gamma(K \rightarrow$ $e v \gamma) / d E_{\gamma} / \Gamma(K \rightarrow \mu v)$ for photon energies $10<E_{\gamma}<250 \mathrm{MeV}$.

We are presently finalizing new determinations of the $K_{L}$ and $K_{S}$ lifetimes using the whole KLOE data set, consisting of more than $10^{9} \phi \rightarrow K_{S} K_{L}$ decays.
\end{abstract}

35th International Conference of High Energy Physics - ICHEP2010,

July 22-28, 2010

Paris France

${ }^{*}$ Speaker.

${ }^{\dagger}$ F. Ambrosino, A. Antonelli, M. Antonelli, F. Archilli, C. Bacci, M. Barva, P. Beltrame, G. Bencivenni, S. Bertolucci, C. Bini, C. Bloise, S. Bocchetta, V. Bocci, F. Bossi, D. Bowring, P. Branchini, S. A. Bulychjov, R. Caloi, P. Campana, G. Capon, D. Capriotti, T. Capussela, G. Carboni, F. Ceradini, F. Cesario, F. Cervelli, S. Chi, G. Chiefari, P. Ciambrone, F. Crucianelli, S. Conetti, E. De Lucia, A. De Santis, P. De Simone, G. De Zorzi, S. Dell'Agnello, A. Denig, A. Di Domenico, C. Di Donato, S. Di Falco, B. Di Micco, A. Doria, M. Dreucci, A. Farilla, G. Felici, A. Ferrari, M. L. Ferrer, G. Finocchiaro, S. Fiore, C. Forti, P. Franzini, C. Gatti, P. Gauzzi, S. Giovannella, E. Gorini, E. Graziani, M. Incagli, W. Kluge, M. Jacewicz, V. Kulikov, F. Lacava, G. Lanfranchi, J. Lee-Franzini, D. Leone, M. Martemianov, M. Martini, P. Massarotti, M. Matsyuk, W. Mei, S. Meola, R. Messi, S. Miscetti, M. Moulson, S. Müller, F. Murtas, M. Napolitano, F. Nguyen, M. Palutan, E. Pasqualucci, L. Passalacqua, A. Passeri, V. Patera, F. Perfetto, L. Pontecorvo, M. Primavera, P. Santangelo, E. Santovetti, G. Saracino, R. D. Schamberger, B. Sciascia, A. Sciubba, F. Scuri, I. Sfiligoi, A. Sibidanov, T. Spadaro, E. Spiriti, M. Tabidze, C. Taccini, M. Testa, L. Tortora, P. Valente, B. Valeriani, G. Venanzoni, S. Veneziano, A. Ventura, S. Ventura, R.Versaci, I. Villella, G. Xu. 
Introduction The KLOE experiment collected $\sim 2.5 \mathrm{fb}^{-1}$ of integrated luminosity at DAФNE, the Frascati $\phi$-factory [1]. DA $\Phi N E$ is an $e^{+} e^{-}$collider that operates at the $\phi$-meson mass. A $\phi$ factory offers the possibility to select pure kaon beams, the kaon pair is in an antisymmetric state so that the final state is always $K_{S} K_{L}$. Therefore, the detection of a $K_{L}$ signals the presence of a $K_{S}$ of known momentum and direction, independently of its decay mode. The same holds for charged kaons. The KLOE detector consists of a large cylindrical drift chamber, surrounded by a lead/scintillating-fiber sampling calorimeter. A superconducting coil surrounding the calorimeter provides a $0.52 \mathrm{~T}$ magnetic field.

CKM unitarity The most precise test of CKM unitarity is given by $\left|V_{\mathrm{ud}}\right|^{2}+\left|V_{\mathrm{us}}\right|^{2}+\left|V_{\mathrm{ub}}\right|^{2}=1$ where $\left|V_{\mathrm{ud}}\right|$ is measured from superallowed $0^{+} \rightarrow 0^{+}$nuclear $\beta$ decay, $\left|V_{\mathrm{us}}\right|$ extracted from kaon semileptonic decay and $\left|V_{\mathrm{ub}}\right|$ being negligible. The kaon semileptonic decay rate is given by $\operatorname{BR}(K \rightarrow \pi \ell v(\gamma)) / \tau=G^{2} m_{K}^{5} /\left(768 \pi^{3}\right) C_{K}^{2}\left|V_{\mathrm{us}}\right|^{2}\left|f_{+}(0)\right|^{2} I_{K}^{\ell} S_{\mathrm{ew}}\left[1+\delta_{\mathrm{SU}(2)}+\delta_{\mathrm{em}}\right]$, where the branching rations and the lifetimes are obtained experimentally from semileptonic decays inclusive of radiation. The theoretical inputs are: the universal short distance electroweak correction $S_{\mathrm{ew}}$, the hadronic form factor at zero momentum transfer $f_{+}(0)$, the form factor correction for strong $\mathrm{SU}(2)$ breaking $\delta_{\mathrm{SU}(2)}$ and the long distance electromagnetic effects $\delta_{\mathrm{ew}}$. KLOE has measured all relevant inputs for charged and neutral kaons: all the branching ratios, lifetimes and form factors. The measured values of $V_{\text {us }} f_{+}(0)$ are [2]: 0.2155(7) for $K_{L} e 3,0.2167(9)$ for $K_{L} \mu 3,0.2153(14)$ for $K_{S} e 3,0.2152(13)$ for $K^{ \pm} e 3$, and $0.2132(15)$ for $K^{ \pm} \mu 3$ decays. Their average yields $V_{\mathrm{us}} f_{+}(0)=$ $0.2157(6)\left(\chi^{2} / \mathrm{ndf}=7.0 / 4\right.$, Prob $\left.=13 \%\right)$, with $0.28 \%$ accuracy to be compared with the $0.23 \%$ of the world average $V_{\mathrm{us}} f_{+}(0)=0.2166(5)$ [3]. Defining $r_{\mu e}=\left|V_{\mathrm{us}} f_{+}(0)\right|_{\mu 3}^{2} /\left|V_{\mathrm{us}} f_{+}(0)\right|_{e 3}^{2}=g_{\mu}^{2} / g_{e}^{2}$, where $g_{\ell}$ is the coupling strength at the $W \rightarrow \ell v$ vertex, lepton universality can be tested comparing the measured value with the Standard Model prediction $r_{\mu e}^{S M}=1$. KLOE obtained $r_{\mu e}=1.000(8)$, averaging between charged and neutral modes, to be compared with $\left(r_{\mu e}\right)_{\pi}=1.0042(33)$ from leptonic pion decays [4], and $\left(r_{\mu e}\right)_{\tau}=1.0005(41)$ from leptonic $\tau$ decays [5]. Using $V_{\mathrm{us}} f_{+}(0)$ from $K_{\ell 3}$ decays and $f_{+}(0)=0.964(5)$ [6], we obtain $V_{\text {us }}=0.2237(13)$. Moreover $V_{\text {us }} / V_{\text {ud }}$ can be measured using the ratio of the radiative inclusive decay rates of $K^{ \pm} \rightarrow \mu^{ \pm} v(\gamma)$ and $\pi^{ \pm} \rightarrow \mu^{ \pm} v(\gamma)$, combined with the lattice calculation of $f_{K} / f_{\pi}$ [7]. Using $\operatorname{BR}\left(K^{ \pm} \rightarrow \mu^{ \pm} v\right)=0.6366(17)$ from KLOE [8] and $f_{K} / f_{\pi}=1.189(7)$ [9], we get $V_{\mathrm{us}} / V_{\mathrm{ud}}=0.2323(15)$. Combining this result with $V_{\text {us }}$ from $K_{\ell 3}$ decays and $V_{\text {ud }}=0.97418(26)$ [10], CKM unitary has been tested to a $0.6 \sigma$ level: $1-\left|V_{\mathrm{ud}}\right|^{2}+\left|V_{\mathrm{us}}\right|^{2}+\left|V_{\mathrm{ub}}\right|^{2}=4(7) \times 10^{-4}$. We then obtained $G_{\mathrm{CKM}}=G_{\mathrm{F}} \sqrt{\left|V_{\mathrm{ud}}\right|^{2}+\left|V_{\mathrm{us}}\right|^{2}+\left|V_{\mathrm{ub}}\right|^{2}}=$ $1.16633(35) \times 10^{-5} \mathrm{GeV}^{-2}$, with $G_{F}=1.166371(35) \times 10^{-5} \mathrm{GeV}^{-2}$. At present, this result is more accurate than what obtained from $\tau$ decays and electroweak precision tests.

Measurement of $R_{K}=\Gamma(K e 2) / \Gamma(K \mu 2)$ The decay $K^{ \pm} \rightarrow e^{ \pm} v$ is strongly suppressed in SM because of the helicity conservation and represents a powerful probe for new physics effects. Small deviations from SM value of $R_{K}$, evaluated with high precision, are expected within MSSM with lepton flavour violation by the charged Higgs [11]. $R_{K}$ has been measured with a $1.3 \%$ accuracy, using the complete KLOE data set: $R_{K}=\left(2.493 \pm 0.025_{\text {stat }} \pm 0.019_{\text {syst }}\right) \times 10^{-5}$ [12]. The effect of radiative corrections has been carefully evaluated in a dedicated measurement. The result of $\left(\mathrm{d} \Gamma(K \rightarrow e v \gamma) / \mathrm{d} E_{\gamma}\right) / \Gamma(K \rightarrow \mu v)$ integrated over $10<E_{\gamma}<200 \mathrm{MeV}$ is $R_{\gamma}=\left(1.483 \pm 0.066_{\text {stat }} \pm\right.$ $\left.0.013_{\text {syst }}\right) \times 10^{-5}[12]$ in agreement with the $\chi \mathrm{Pt}$ prediction at $\mathscr{O}\left(e^{2} p^{4}\right)$. Our result on $R_{K}$ gives 
strong constraints for $\tan \beta$ and $m_{H^{ \pm}}$, as a function of the 1-3 slepton-mass matrix element $\Delta_{13}$.

$K_{L}$ lifetime measurement The $K_{L}$ lifetime $\left(\tau_{L}\right)$ uncertainty is the limiting factor on the accuracy of $V_{\text {us }}$ measured from $K_{L}$ semileptonic decays. To improve this accuracy the KLOE collaboration has decided to perform a new $\tau_{L}$ measurement with $\sim 46$ million of $K_{L} \rightarrow 3 \pi^{0}$ events. $K_{L}$ mesons are tagged by detecting $K_{S} \rightarrow \pi^{+} \pi^{-}$decays and the proper time distribution obtained with $K_{L} \rightarrow 3 \pi^{0}$ decays is used to measure $K_{L}$ lifetime, exploiting the high branching ratio of $3 \pi^{0}$ decay mode and its high detection efficiency. The preliminary result is: $\tau_{L}=$ $50.56 \pm 0.14_{\text {stat }} \pm 0.21_{\text {syst }}$ ns [13] compatible with the previous KLOE measurement [14]. The expected statistical uncertainty with the whole data sample is $\sigma_{\text {stat }} \sim 0.1 \mathrm{~ns}$, and the systematic contribution, partially statistical in nature, is expected to decrease as well.

$K_{S}$ lifetime measurement KLOE measures $K_{S}$ lifetime $\left(\tau_{S}\right)$ with pure $K_{S}$ beam and exploiting the event by event knowledge of $K_{S}$ momentum. The lifetime is measured by fitting the proper time $\left(t^{*}\right)$ distribution of $K_{S} \rightarrow \pi^{+} \pi^{-}$decays. The $t^{*}$ resolution is improved reconstructing the interaction point event-by-event using a geometrical fit, selecting events with pions decaying at large angle with respect to the $K_{S}$ direction. The efficiency of the selection is $\sim 13 \%$. Since the resolution depends on the $K_{S}$ direction, we fit the proper time distribution from -1 to $6.5 \tau_{S}$ in each of 180 bins in $\cos \left(\theta_{K}\right)$ and $\phi_{K}$. The statistical error on $\tau_{s}$ is less than $0.1 \%$. The final result has now been submitted for preliminary [15]: $\tau_{S}=\left(89.562 \pm 0.029_{\text {stat }} \pm 0.043_{\text {syst }}\right)$ ps.

\section{References}

[1] F. Bossi, E. De Lucia, J. Lee-Franzini, S. Miscetti, M. Palutan (KLOE), Riv. Nuovo Cim. 031, 531 (2008), 0811.1929

[2] F. Ambrosino et al. (KLOE), JHEP 04, 059 (2008), 0802 . 3009

[3] M. Antonelli et al. (FLAVIAnet working group on kaon decays) (2008), 0801.1817

[4] M.J. Ramsey-Musolf, S. Su, S. Tulin, Phys. Rev. D76, 095017 (2007), 0705 . 0028

[5] M. Davier, A. Hocker, Z. Zhang, Rev. Mod. Phys. 78, 1043 (2006), hep-ph / 0507078

[6] UKQCD/RBC Collaboration, Phys. Rev. Lett. 100, 141601 (2008)0710 . 5136

[7] W.J. Marciano, Phys. Rev. Lett. 93, 231803 (2004), hep-ph / 0402299

[8] F. Ambrosino et al. (KLOE), Phys. Lett. B632, 76 (2006)

[9] HPQCD and UKQCD Collaborations, Phys. Rev. Lett. 100, 062002 (2008)

[10] J.C. Hardy, I.S. Towner, Phys. Rev. C79 (2009) 055502

[11] A. Masiero, P. Paradisi, R. Petronzio, Phys. Rev. D74, 011701 (2006), hep-ph / 0511289

[12] F. Ambrosino et al. (KLOE), Eur. Phys. J. C64, 627 (2009), 0907 . 3594

[13] S. Bocchetta et al. (KLOE), PoS KAON09 006 (2009)

[14] F. Ambrosino et al. (KLOE), Phys. Lett. B 626 (2005) 15 hep-ex/ 0507088

[15] M. Dreucci et al. (KLOE), submitted to EPJC 1011.2668 